\title{
Supervivencia de los trabajadores de salud infectados por SARS-CoV-2 en el contexto de la vacunación contra la COVID-19 en el Perú
}

\section{Survival of health workers infected by SARS-CoV-2 in the context of vaccination against COVID-19 in Peru}

\author{
Stefan Escobar-Agreda ${ }^{1,2, a}$, Javier Silva-Valencia ${ }^{1,2, b}$, Leonardo Rojas-Mezarina ${ }^{1,2, c}$, Javier Vargas-Herrera ${ }^{1,2, d}$ \\ 1 Instituto Nacional de Salud. Lima, Perú. \\ ${ }^{2}$ Unidad de Telesalud, Facultad de Medicina, Universidad Nacional Mayor de San Marcos. Lima, Perú. \\ ${ }^{a}$ Médico cirujano, ORCID: https://orcid.org/0000-0002-8355-4310 \\ ${ }^{b}$ Médico cirujano, magister en informática biomédica y salud global. ORCID: https://orcid.org/0000-0002-5982-2821 \\ ${ }^{\circ}$ Médico cirujano, ORCID: https://orcid.org/0000-0003-0293-7107 \\ ${ }^{d}$ Médico cirujano, magister en epidemiologia. ORCID: https://orcid.org/0000-0002-1910-602X
}

Correspondencia:

Javier Vargas Herrera

jvargash@unmsm.edu.pe

Recibido: 16 de julio 2021

Aprobado: 12 de agosto 2021

Publicación en línea: 14 de agosto 2021

Conflictos de interés: Los autores declaran no tener conflictos de interés.

Fuente de financiamiento: Instituto Nacional de Salud

Citar como: Escobar-Agreda S, Silva-Valencia J, Rojas-Mezarina L, Vargas-Herrera J. Supervivencia de los trabajadores de salud infectados por SARS-CoV-2 en el contexto de la vacunación contra la COVID-19 en el Perú. An Fac med. 2021;82(2):106-12. DOI: https://doi.org/10.15381/anales. v82i2.20766

\section{An Fac med. 2021;82(2):106-12. / DOI: https://doi.org/10.15381/anales.v82i2.20766.}

Resumen

Objetivos. Evaluar la supervivencia de los trabajadores de salud infectados por SARS-CoV-2 en el contexto del proceso de vacunación contra la COVID-19 en el Perú. Métodos. Se realizó un análisis de supervivencia a partir de datos provenientes de las bases nacionales en salud. Se incluyó datos de personas entre 18 y 59 años infectadas por SARS-CoV-2 evidenciada por prueba molecular o antigénica. Se elaboraron gráficos de Kaplan Meier para comparar la sobrevida de los trabajadores de salud y el resto de la población durante el año 2021 y el trabajador de salud durante la primera y segunda ola de mortalidad en el Perú en el 2020 y 2021, respectivamente. Resultados. Se incluyeron datos de 998295 personas. La edad promedio fue 41,2 años (DE 15,8) y 485167 (48,6\%) fueron mujeres. Se encontró un mayor nivel de sobrevida de los trabajadores de salud después de la vacunación con respecto a la población en general y a la población de los trabajadores de salud antes de la vacunación. Se evidenció que, al inicio de la segunda ola, el riesgo de morir de los trabajadores de salud era el doble del que tenían en la primera ola $(H R=2)$. Después de la vacunación (en el sexto mes de la segunda ola), el riesgo de morir disminuyó hasta 87,5\% menos que en la primera ola $(H R=0,125)$. Conclusiones. Se ha evidenciado un cambio positivo en el nivel de sobrevida de los trabajadores de salud infectado por SARS-CoV-2 durante el contexto de la vacunación contra la COVID-19 en el Perú.

Palabras clave: Sobrevida; Trabajadores de Salud; Vacunación; COVID-19; Perú (fuente: DeCS BIREME).

\section{Abstract}

Objectives. To evaluate the survival of health personnel infected by SARS-CoV-2 in the context of the vaccination process against COVID-19 in Peru. Methods: A survival analysis was performed using data from national health databases. Data from people between 18 and 59 years old infected with SARS-CoV-2 as evidenced by molecular or antigenic tests were included. Kaplan Meier graphs were produced to compare the survival of health personnel and the rest of the population during 2021 and health personnel during the first and second wave of mortality in Peru in 2020 and 2021, respectively. Results. Data from 998295 people were included. The average age was 41.2 years (SD 15.8) and 485167 (48.6\%) were women. A higher level of survival of health workers after vaccination was found compared to the general population and to the population of health workers before vaccination. It was evidenced that, at the beginning of the second wave, the risk of dying for health workers was twice that of the first wave $(H R=2)$. After vaccination (in the sixth month of the second wave), the risk of dying decreased to $87.5 \%$ less than in the first wave (HR $=0.125)$. Conclusions. A positive change has been evidenced in the level of survival of health personnel infected by SARS-CoV-2 during the context of vaccination against COVID-19 in Peru.

Keywords: Survival; Health Personnel; Vaccination; COVID-19; Peru (source: MeSH NLM). 


\section{INTRODUCCIÓN}

LA COVID-19, enfermedad causada por el virus SARS-CoV-2, ha generado un impacto importante en la salud de la población mundial no solo por su capacidad de diseminarse sino además por el riesgo de causar la muerte ${ }^{(1)}$. Actualmente, Perú constituye uno de los países con la mayor tasa de mortalidad por esta enfermedad a nivel mundial (2). En este contexto, el gobierno peruano aprobó el Plan Nacional de Vacunación contra la COVID-19, el 16 de octubre de $2020^{{ }^{(3)}}$ e inició su implementación el 9 de febrero del año 2021, dado su reconocimiento como uno de los principales recursos para combatir la pandemia a nivel poblacional ${ }^{(4)}$. El esquema del plan de vacunación contra la COVID-19 en el Perú priorizó la vacunación de los trabajadores de salud al ser una de las poblaciones más expuestas a este virus ${ }^{(3)}$. Tan sólo en los primeros 100 días de la pandemia se infectaron 1867 médicos, de los cuales murieron $65^{(5)}$.

Algunos reportes a nivel nacional han evaluado de forma preliminar el potencial efecto del proceso de vacunación en el trabajador de salud, evidenciando una reducción en las tasas de hospitalización y mortalidad del personal médico durante los primeros meses del periodo de vacunación en el Perú (6). Sin embargo, hasta la fecha aún no se conoce la variación en el nivel de sobrevida de los trabajadores de salud infectados por el SARSCoV-2, durante el periodo de vacunación, en comparación con el año pasado o respecto al resto de la población adulta. Al momento de la realización del presente análisis, la población adulta aún no había sido vacunada, según el esquema nacional planteado ${ }^{(3)}$. Adicionalmente resulta importante conocer si estas comparaciones varían según el sexo, dada la evidencia que señala la diferencia de riesgo de mortalidad por COVID-19 entre estos subgrupos $(7,8)$.

En el Perú, existen diversos sistemas de información en salud de alcance nacional que incluyen la información nominal sobre las personas que se sometieron a pruebas de laboratorio para el diagnóstico de COVID-19 (NETLAB y SICOVID), trabajadores de salud asistencial y administrativo (INFORHUS) y personas fa- llecidas (SINADEF). Sobre la base de esta información el presente estudio tuvo como objetivo evaluar la sobrevida de los trabajadores de salud infectados por SARS-CoV-2 durante el contexto del proceso de vacunación contra la COVID-19 en el Perú.

\section{MÉTODOS}

\section{Diseño del estudio}

Se realizó un análisis secundario de datos utilizando las bases de datos de SINADEF (registro de fallecidos), INFORHUS (registro de trabajadores de salud), SICOVID (registro de pruebas de antígeno para el diagnóstico de SARS-Cov-2) y NETLAB (registro de pruebas moleculares para el diagnóstico molecular de SARS-Cov-2), las cuales contienen información sobre las personas evaluadas para determinar su estado de infección por el virus SARSCoV-2 mediante pruebas moleculares o pruebas antigénicas, ocupación para la clasificación como trabajadores de salud, y personas fallecidas a nivel nacional.

\section{Población}

Se incluyeron observaciones de personas entre 18 a 59 años con una prueba positiva a SARS-CoV-2 evidenciada mediante prueba molecular (PM) o prueba antigénica ( $P A$ ) registradas en las bases de datos NETLAB o SICOVID hasta el 30 de junio de 2021; en el caso de personas con más de una prueba, se consideró sólo a la última prueba. Se excluyó a aquellas observaciones que no permitían la identificación de los sujetos, la fecha de obtención de la muestra posterior a la fecha de defunción, o cuya fecha de defunción fue posterior a los 60 días a partir de la fecha de obtención de la muestra (para la prueba molecular o antigénica). La probabilidad de que una muerte se atribuya a la COVID-19 después de los 60 días del diagnóstico de la enfermedad es muy baja ${ }^{(9)}$. Con el fin de obtener estimados a nivel nacional no se realizó un muestreo y se incluyeron todas las observaciones que cumplieron con los criterios de selección. En base a ello se definieron en 2 subpoblaciones de estudio:

Trabajador de salud y población en
general con prueba positiva para
SARS-CoV-2 por PM o PA en el Perú, desde el $1^{\circ}$ de enero al 30 de junio del 2021.

- Trabajador de salud con prueba positiva para SARS-CoV-2 por PM durante la primera y segunda ola de mortalidad en el Perú, en los años 2020 y 2021, respectivamente.

\section{Variable desenlace}

Se categorizó como "fallecido" a los sujetos registrados en la base de datos del SINADEF y cuya defunción haya ocurrido hasta el 30 de junio de 2021 y como "no fallecido" al resto. Adicionalmente se incluyó la fecha de fallecimiento para determinar el tiempo de sobrevida.

\section{Variables de exposición}

\section{Trabajador de salud}

Se categorizó como "trabajador de salud" a las personas registradas en la base de datos INFORHUS actualizada hasta el 31 de enero del 2021. Con el fin de optimizar esta clasificación se añadió también como trabajadores de salud a aquellas personas que no estaban registradas en INFORHUS que hayan reportado que tiene una ocupación como trabajadores de salud: "médico", "enfermero", "obstetra", entre otros, en el momento de la obtención de la muestra.

\section{Ola de COVID-19}

Se categorizó a aquellos pacientes que hayan sido infectados por SARSCoV-2 durante la primera o segunda ola de mortalidad de COVID-19 en el Perú. El inicio de cada ola se definió como el primer día de la semana epidemiológica en la cual ocurrió un incremento aproximado del $75 \%$ de fallecimientos respecto al promedio histórico de muertes según los datos de SINADEF. Se consideró como inicio de la primera ola el 19 de abril de 2020 y como el inicio de la segunda ola el 3 de enero de 2021. Dada la disponibilidad de los datos, se consideró un tiempo de análisis de 180 días para cada ola.

\section{Covariables}

Entre las covariables se incluyeron sexo categorizado como masculino y femenino, edad evaluada en años, contar o no con seguro de salud, y pertenecer o no a la región Lima. 


\section{Análisis estadístico}

El análisis de datos se hizo en el paquete estadístico Stata v16.0 para Windows. Inicialmente se realizó un análisis descriptivo para evaluar las características de los trabajadores de salud y la población en general infectadas por SARSCoV-2 incluidas en el estudio. Para el análisis de supervivencia se elaboraron curvas de Kaplan Meier para comparar la sobrevida de los trabajadores de salud y la población en general infectada por SARS-CoV-2 durante el año 2021; y la sobrevida de los trabajadores de salud en la primera y segunda ola de mortalidad dadas durante la pandemia de COVID-19 en el Perú en los años 2020 y 2021. Para esta última comparación, se estimaron los cocientes de riesgos (Hazard ratios, HR) y las diferencias de riesgos (reducción absoluta del riesgo, RAR) de la mortalidad entre ambas olas, con un intervalo de confianza al 95\%. Para ello se emplearon regresiones de Cox y modelos polinomiales con el fin de graficar la evolución de estos indicadores durante el periodo de estudio de ambas olas.

Para mejorar la visualización de la sobrevida de las poblaciones estudiadas en el periodo de estudio, se definieron algunos hitos importantes correspondientes a la fecha del inicio de la vacunación ( 9 de febrero del 2021), inicio de la aplicación segunda dosis ( $1^{\circ}$ de marzo del 2021), e inicio del periodo de protección (14 días después del inicio de la segunda dosis). Este último periodo fue definido en base al momento de la protección sugerido para la vacuna de Sinopharm utilizada para la vacunación a trabajadores de salud en el Perú ${ }^{(10)}$.

\section{Aspectos éticos}

El estudio tuvo la aprobación del Comité de Ética en Investigación de la Facultad de Medicina de la Universidad Nacional Mayor de San Marcos. El estudio se basó en un análisis secundario de los datos contenidos en las bases de datos de los sistemas de información de rutina del Instituto Nacional de Salud y el Ministerio de Salud. El acceso y uso de la información de las bases de datos analizadas fue aprobado mediante cartas de autorización de los titulares de las instituciones donde pertenecen los bancos de datos, en cumplimiento de las competencias del
Instituto Nacional de Salud, en el marco de la vigilancia de la salud pública y en el contexto de la atención de la pandemia de la COVID-19 en el Perú.

\section{RESULTADOS}

Se estudiaron 998295 personas entre 18 a 59 años, cuya última prueba mole- cular o antigénica resultó positiva a SARSCoV-2 desde el inicio de la pandemia. La edad promedio fue 41,2 años (DE: 15,8), 485167 (48,6\%) fueron mujeres, 466459 $(46,7 \%)$ procedían de Lima, 34424 (3,5\%) eran trabajadores de salud y 35981 (3,6\%) había fallecido de acuerdo con su registro en SINADEF. La tabla 1 muestra las características entre el trabajador de salud y el resto de la población incluida en el estudio.

Tabla 1. Características de pacientes adultos entre 18 a 59 años, positivos a COVID-19 por prueba molecular o antigénica en el Perú ( $n=998$ 295), hasta el 30 de junio 2021.

\begin{tabular}{|c|c|c|}
\hline Características & $\begin{array}{l}\text { Trabajador de salud, n (\%) } \\
\text { n=34 } 424\end{array}$ & $\begin{array}{l}\text { No trabajador de salud, } n(\%) \\
\qquad n=963871\end{array}$ \\
\hline Edad (años) & $40 \pm 10,9$ & $41,3 \pm 16$ \\
\hline \multicolumn{3}{|l|}{ Sexo } \\
\hline Femenino & $22502(65,4)$ & $462665(48)$ \\
\hline Masculino & $11922(34,6)$ & $501206(52)$ \\
\hline \multicolumn{3}{|l|}{ Procedencia } \\
\hline No Lima & $13616(39,6)$ & $452843(47)$ \\
\hline Lima & $20808(60,5)$ & $511028(53)$ \\
\hline \multicolumn{3}{|l|}{ Fallecimiento* } \\
\hline No fallecido & 34071 (99) & $928243(96,3)$ \\
\hline Fallecido & $353(1)$ & $35628(3,7)$ \\
\hline
\end{tabular}

*Base de datos de SINADEF

Figura 1. Supervivencia de los trabajadores de salud y el resto de la población infectada por SARSCoV-2, durante la pandemia de COVID-19 en el Perú, 2021 (N=998,292).

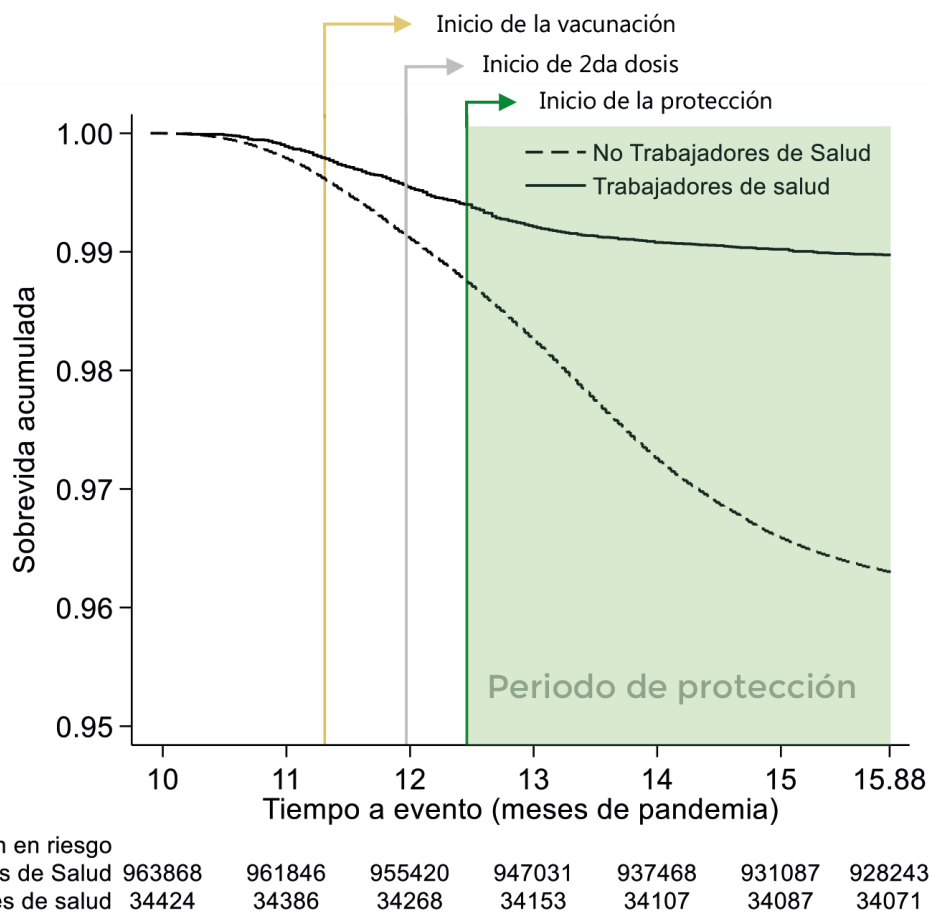


Figura 2. Supervivencia de los trabajadores de salud infectados por SARS-CoV-2, durante la primera y segunda ola de COVID-19 en el Perú, 2020-2021. ( $N=32,159)$

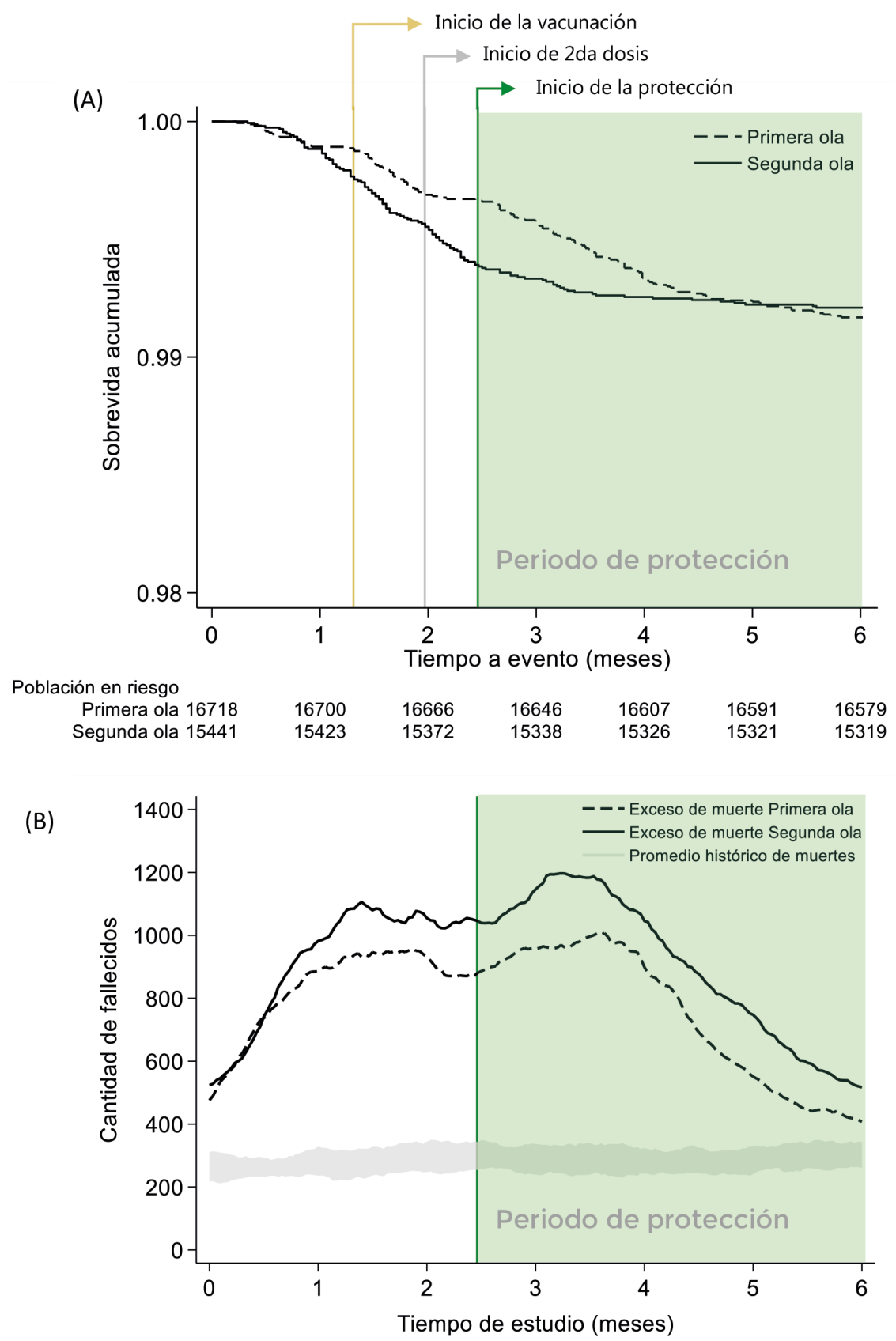

(A) Niveles de sobrevida (Kaplan Meier) de los trabajadores de salud durante la primera y segunda ola de COVID-19 en el Perú. (B) Exceso de mortalidad respecto al promedio histórico de muertes (2017-2019) durante la primera y segunda ola de COVID-19 en el Perú.

\section{Supervivencia del trabajador de salud en relación a la población general}

Las curvas de Kaplan Meier evidenciaron una diferencia en la supervivencia de los trabajadores de salud infectados con el virus de SARS-CoV-2 con respecto al resto de la población general positiva a SARS-CoV-2 en el Perú. Adicionalmente, se evidenció una acentuación de esta diferencia debido al aplanamiento de la supervivencia en el trabajador de salud a partir del 15 de marzo del 2021 (día 374 de la pandemia), fecha correspondiente a los 14 días después del inicio de la aplicación de la segunda dosis. (Figura 1).

Supervivencia del trabajador de salud en la primera y segunda ola de la pandemia COVID-19

Se evidenció también una mayor mortalidad de los trabajadores de salud durante la segunda ola en comparación con la primera. Sin embargo, en los días posteriores, la curva de supervivencia durante la segunda ola mostró un aplanamiento, específicamente a partir del inicio del periodo de protección, dos semanas después del momento en que empezó la aplicación de la segunda dosis (15 de marzo 2021), llegando a disminuir la brecha de supervivencia respecto a la primera ola, según se muestra en la figura 2.

De forma específica, se observó inicialmente una diferencia positiva del riesgo de mortalidad de la segunda ola con respecto a la primera (RAR $>0$ ), lo que indica que el riesgo de morir en la segunda ola fue mayor que en la primera ola, que se fue reduciendo hasta anularse hacia el final del periodo de estudio $(R A R=0)$; así, después de la vacunación, el riesgo de morir de la segunda ola se igualó al de la primera ola. La reducción absoluta del riesgo en el sexto mes de la segunda ola fue menor que cero en comparación con la primera ola ( $R A R<0)$; es decir, el riesgo de morir de los trabajadores de salud fue menor en la segunda ola, en comparación con la primera. (Figura 3).

El cociente de riesgos (Hazard ratio) evidenció una mayor incidencia de mortalidad en la segunda ola con respecto a la primera. El riesgo de morir de los trabajadores de la salud en la segunda ola fue dos veces el riesgo morir en la primera ola $(H R=2)$, antes del inicio de la vacunación. Al iniciar el periodo de protección de la vacunación, el riesgo de morir en la segunda ola se redujo hasta lo similar al riesgo de morir en la primera ola $(H R=1)$. Finalmente, a los seis meses de la segunda ola y después de tres meses y medio del inicio de la protección de la vacuna- 
Figura 3. Diferencias del Riesgo de muerte (Reducción Absoluta de Riesgo, RAR) entre la segunda y primera ola de la COVID-19 en trabajadores de salud infectados por SARS-CoV-2 en el Perú, 2020-2021. ( $N=32,159)$

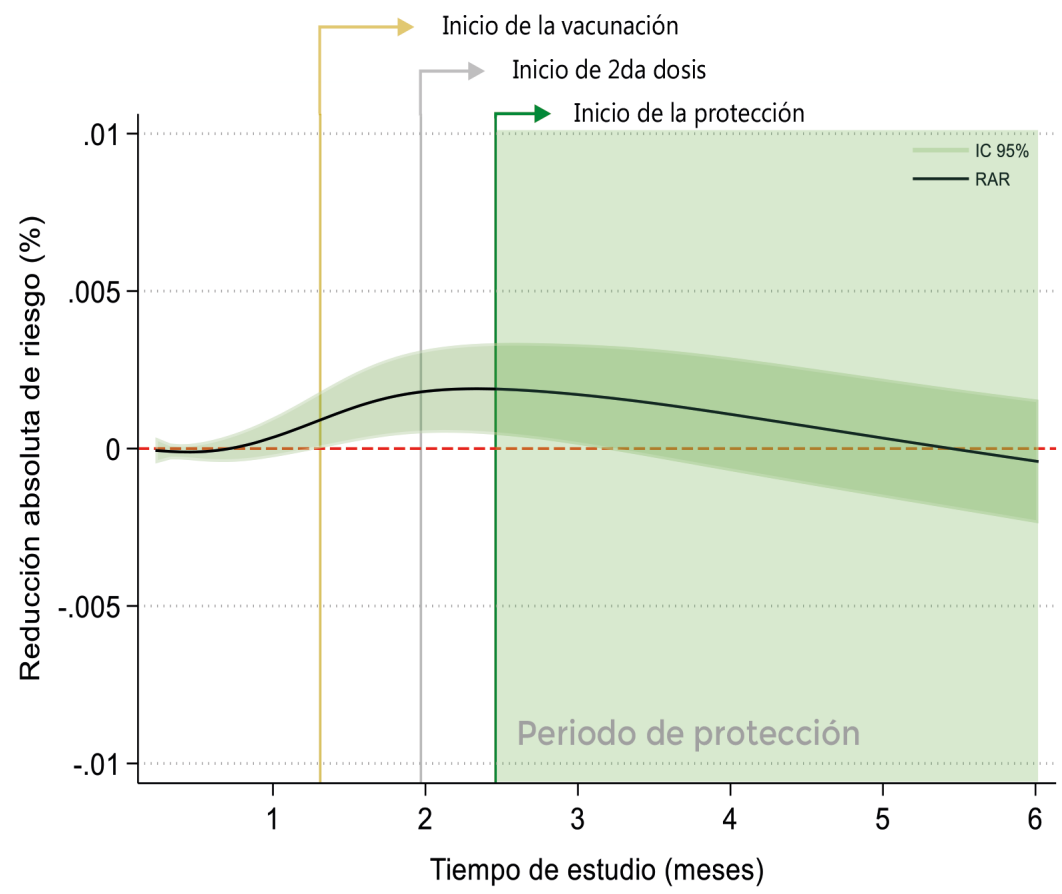

Abreviaturas: RAR = Reducción absoluta de riesgo; IC 95\%: Intervalo de confianza al 95\% ción, el riesgo de morir en la segunda ola fue $87,5 \%$ menor en comparación con la primera ola $(H R=0,125)$. (Figura 4$)$.
Al realizar la comparación de las curvas de mortalidad de los trabajadores de salud durante la primera ola y durante
Figura 4. Cocientes del riesgo de muerte (Hazard Ratio) entre la segunda y primera ola la COVID-19 en trabajadores de salud infectados por SARS-CoV-2 en el Perú, 2020-2021. (N=32,159)

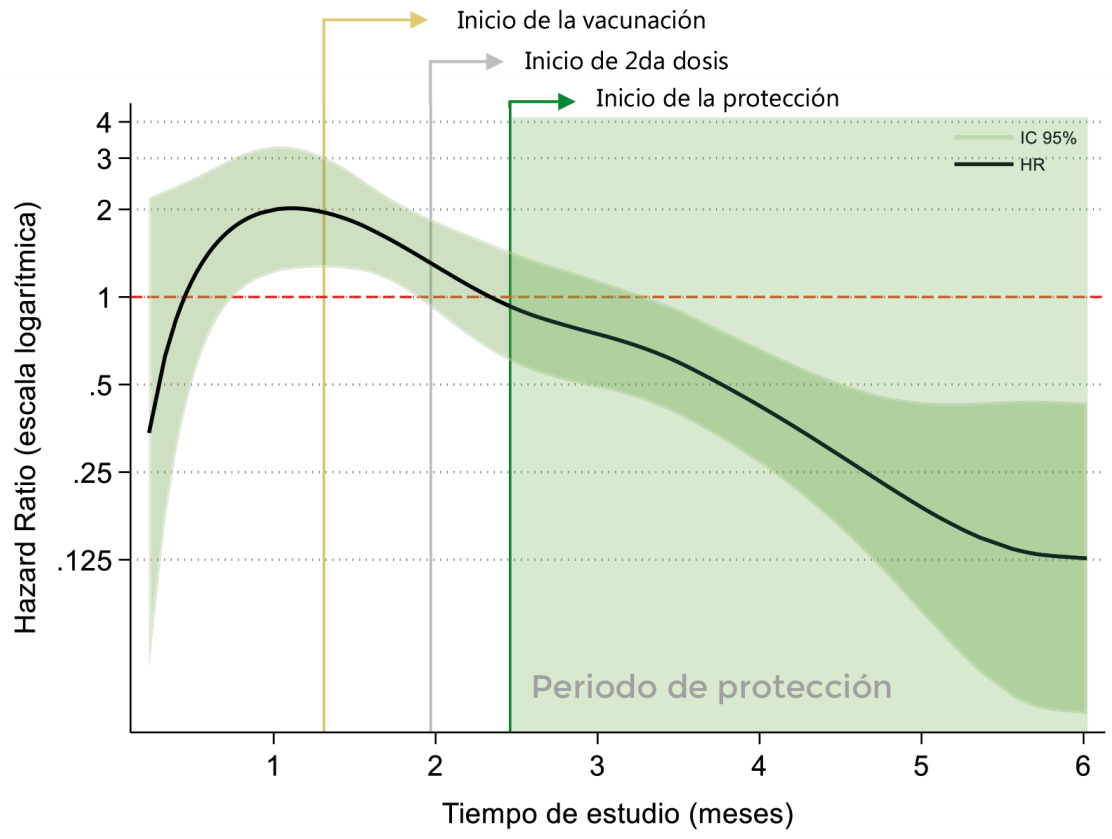

Abreviaturas: HR = Hazard ratio; IC 95\%: Intervalo de confianza al 95\% la segunda ola, por sexos, se evidenció también un aplanamiento de curva de supervivencia durante la segunda ola, a partir del inicio del periodo de protección. Sin embargo, esta fue mucho más evidente entre el trabajador de salud masculino. (Figuras 5 y 6 ).

\section{DISCUSIÓN}

Los resultados del presente estudio muestran de forma preliminar el cambio positivo de la sobrevida de los trabajadores de salud en el contexto de la primera fase de vacunación contra la COVID-19 en el Perú. Estos resultados mostraron de forma consistente que, después del periodo de vacunación, la población de los trabajadores de salud mejoró su sobrevida en comparación con la sobrevida de la población en general que no es trabajador de salud; y que el trabajador de salud mejoró su sobrevida durante la segunda ola de trasmisión de la COVID-19 en el año 2021, en comparación con su sobrevida durante la primera ola en el año 2020.

Se observó un mayor nivel de sobrevida de los trabajadores de salud respecto a la población en general infectada por SARS-CoV-2 durante la primera mitad del año 2021. Esta situación inicialmente podría explicarse por un mayor acceso a los servicios de salud, mayor nivel socioeconómico y mayor conocimiento sobre los cuidados respecto a su enfermedad de los trabajadores de salud respecto a la población general. Sin embargo, el incremento observado en la diferencia de sobrevida entre ambos grupos fue notorio después del periodo que siguió a la vacunación de los trabajadores de salud, lo que se puede atribuir al efecto de esta intervención sobre la mortalidad.

Adicionalmente se evidenció una mayor sobrevida de los trabajadores de salud infectados por SARS-CoV-2 en la segunda ola, después el periodo de vacunación en el Perú, en comparación con la primera ola. En este contexto, se observó una mayor mortalidad de los trabajadores de salud al inicio de la segunda ola, que estaría relacionado con la mayor mortalidad que ocurrió a nivel nacional 
Figura 5. Supervivencia de los trabajadores de salud (sexo masculino) infectados por SARS-CoV-2, durante la primera y segunda ola de COVID-19 en el Perú, 2020-2021 ( N=11,523).

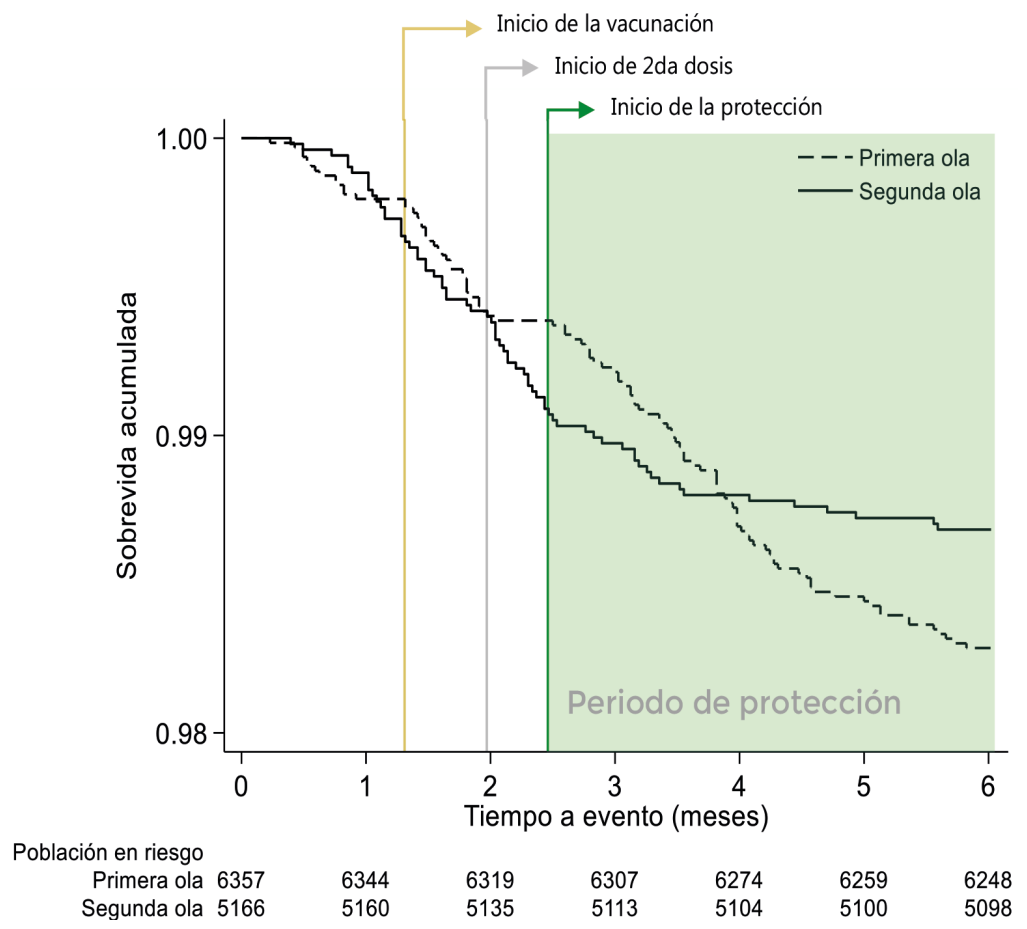

durante el año 2021. Por otro lado, la comparación de riesgos a través de indicadores (RAR, HR) evidenciaron que el

incremento inicial de la mortalidad de la segunda ola tuvo una desaceleración a partir del inicio de la vacunación con un

Figura 6. Supervivencia de las trabajadoras de salud (sexo femenino) infectadas por SARS-CoV-2, durante la primera y segunda ola de COVID-19 en el Perú, 2020-2021 (N=20,636).

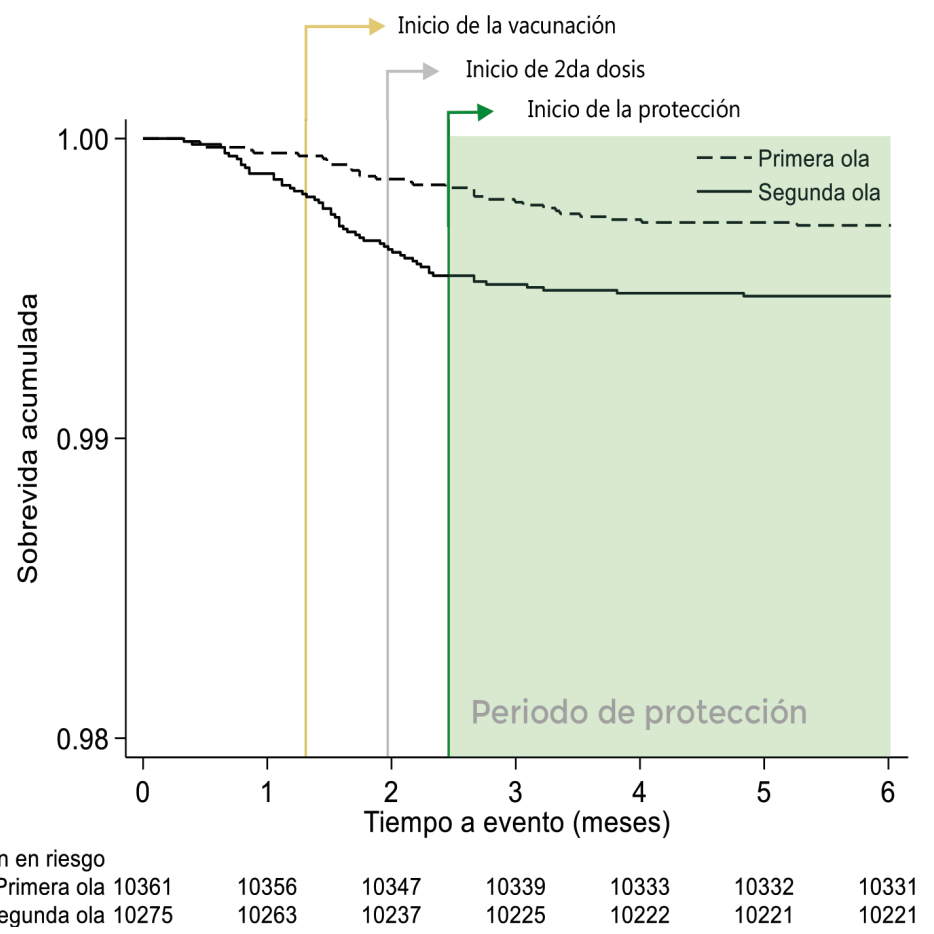

franco descenso a partir del periodo de protección, logrando anular la diferencia de mortalidad entre segunda y primera ola. El riesgo de morir de los trabajadores en la segunda ola, antes de la vacunación, era el doble en comparación con la primera ola, y pasó a ser 87,5\% menor en comparación con la primera ola, después de la vacunación. Esto sugiere un posible impacto de la vacunación sobre la mortalidad desde el inicio de su aplicación y se consolidaría después de la aplicación de la segunda dosis.

Finalmente, en el subanálisis realizado por sexo se evidenció que el trabajador de salud masculino infectado por SARSCoV-2, mostró un mayor cambio en el nivel de sobrevida durante el periodo de vacunación logrando no solo anular la diferencia de riesgo de mortalidad de la segunda ola respecto a la primera, sino superándola hacia el final del periodo de estudio. Esta situación fue menos evidente en el sexo femenino debido a la menor mortalidad en general de este subgrupo ante el COVID-19 ${ }^{(8)}$.

El presente estudio tiene algunas limitaciones. En primer lugar, al no contar con datos nominales de las personas vacunadas a nivel nacional no se puede determinar si todo trabajador de salud incluido fue finalmente vacunado durante el periodo de estudio. Sin embargo, la cobertura de vacunación en el Perú entre los trabajadores de salud fue alta; así, sólo entre los médicos alcanzó el $86,7 \%$ según el vacunómetro del Colegio Médico del Perú ${ }^{(11)}$, por lo tanto, el personal no vacunado sería bajo y su exclusión no modificaría de forma importante los resultados encontrados. Adicionalmente, en la comparación de la sobrevida entre las olas de COVID-19 el uso de datos retrospectivos no permite evaluar con exactitud el pronóstico a 60 días de los pacientes infectados en las últimas semanas del periodo de estudio; sin embargo, este sesgo actuaría atenuando las diferencias de sobrevida encontradas entre la primera y segunda ola, la cual podría ser aún mayor si se incluyesen datos de mortalidad de los pacientes en los siguientes dos meses. Otro aspecto en este análisis es la diferencia en la dis- 
ponibilidad de recursos en salud peruano para enfrentar la pandemia de COVID-19 en la primera y segunda ola. Si bien esta situación ha podido favorecer en la reducción de la mortalidad en la segunda ola, el cambio de sobrevida visualizado durante el inicio de la primera y segunda dosis y el periodo de protección sugieren fuertemente la contribución del proceso de vacunación en el cambio de sobrevida de los trabajadores de salud.

Los resultados encontrados coinciden con las evidencias presentadas a nivel nacional que sugieren una potencial influencia de la vacunación en la tasa de mortalidad en el personal médico, dada la disminución de este indicador durante los primeros meses de la periodo de vacunación en el Perú ${ }^{(6)}$. De la misma manera, esto coincide con la evidencia mostrada en países como Chile, Estados Unidos y el Reino Unido, que han mostrado una marcada reducción en la mortalidad a nivel general conforme el avance el desarrollo de la vacunación contra la COVID-19 en sus respectivos países ${ }^{(12,13,14)}$.

En conclusión, se muestra un cambio positivo en el nivel de sobrevida de los trabajadores de salud durante el proceso de vacunación contra la COVID-19 en el Perú, especialmente en personal de sexo masculino. Este resultado sumado a la evidencia encontrada a nivel nacional e internacional reafirma el valor de la vacunación como una de las principales estrategias para combatir los efectos de la pandemia de COVID-19, especialmente en población con elevada exposición al virus como el trabajador de salud.

Siguiendo esta línea, se recomienda la realización de estudios orientados a estimar el impacto de la vacunación contra la COVID-19 en el Perú sobre la mortalidad de los trabajadores de salud en términos de efectividad, así como la evaluación de otros indicadores de interés como infección, hospitalización, e ingreso a cuidados intensivos, que permitan evaluar su utilidad de forma más precisa tal como se ha realizado en otros países ${ }^{(15,16,17,18)}$.

\section{REFERENCIAS BIBLIOGRÁFICAS}

1. Zhang J, Wang X, Jia X, Li J, Hu K, Chen G, et al. Risk factors for disease severity, unimprovement, and mortality in COVID-19 patients in Wuhan, China. Clin Microbiol Infect. $1^{\circ}$ de junio de 2020;26(6):767-72. DOI: 10.1016/j.cmi.2020.04.012

2. Mortality Analyses [Internet]. Johns Hopkins Coronavirus Resource Center. [citado el 14 de julio de 2021]. Disponible en: https://coronavirus.jhu.edu/ data/mortality

3. Ministerio de Salud del Perú [Internet]. Documento técnico: Plan Nacional de Vacunación contra la COVID-19. RM N848-2020/MINSA, RM N848-2020/ MINSA oct 16, 2020 p. 13-5 [citado el 14 de julio de 2021]. Disponible en: http://www.digesa.minsa. gob.pe/Orientacion/RM-848-2020-MINSA.pdf

4. CDC [Internet]. Benefits of Getting a COVID-19 Vaccine. Centers for Disease Control and Prevention. 2021 [citado el 29 de abril de 2021]. Disponible en: https://www.cdc.gov/coronavirus/2019-ncov/ vaccines/vaccine-benefits.html

5. Galan-Rodas E, Tarazona-Fernandez A, PalaciosCeli M. Riesgo y muerte de los médicos a 100 dias del estado de emergencia por el COVID-19 en Perú. Acta Med Peru. 2020;37(2):119-21. DOI: https://doi. org/10.35663/ amp.2020.372.1033

6. Instituto Nacional de Salud [Internet]. Evidencias preliminares sobre el efecto de vacunación contra la COVID-19 en el Perú. [citado el 14 de julio de 2021]; Disponible en: https://boletin.ins.gob.pe/evidencias_ preliminares_sobre_el_efecto_de_vacunacion/

7. Wei X, Xiao Y-T, Wang J, Chen R, Zhang W, Yang $Y$, et al. Sex Differences in Severity and Mortality Among Patients With COVID-19: Evidence from Pooled Literature Analysis and Insights from Integrated Bioinformatic Analysis. ArXiv200313547 QBio. Disponible en: http://arxiv.org/abs/2003.13547

8. Munayco C, Chowell G, Tariq A, Undurraga EA, Mizumoto K. Risk of death by age and gender from CoVID-19 in Peru, March-May, 2020. Aging. 21 de julio de 2020;12(14):13869-81. DOI: 10.18632/aging.103687

9. Marschner IC. Estimating age-specific COVID-19 fatality risk and time to death by comparing population diagnosis and death patterns: Australian data. BMC
Med Res Methodol. 21 de junio de 2021;21(1):126 DOI: https://doi.org/10.1186/s12874-021-01314-w

10. World Health Organization [Internet]. Annexes to the interim recommendations for use of the inactivated COVID-19 vaccine BIBP developed by China National Biotec Group (CNBG), Sinopharm: grading of evidence: evidence to recommendation tables, 7 May 2021. 2021 [citado el 22 de junio de 2021]. Disponible en: https://apps.who.int/iris/ handle/10665/341254

11. Vacunómetro-CMP [Internet]. Colegio Médico del Perú - Consejo Nacional. [citado el 16 de julio de 2021]. Disponible en: https://www.cmp.org.pe/ vacunometro-cmp/

12. Jara A, Undurraga EA, González C, Paredes F, Fontecilla $T$, Jara $G$, et al. Effectiveness of an Inactivated SARS-CoV-2 Vaccine in Chile. N Engl J Med. 7 de julio de 2021. DOI: 10.1056/NEJMoa2107715

13. Pilishvili T, Fleming-Dutra KE, Farrar JL, Gierke R, Mohr NM, Talan DA, et al. Interim Estimates of Vaccine Effectiveness of Pfizer-BioNTech and Moderna COVID-19 Vaccines Among Health Care Personne - 33 U.S. Sites, January-March 2021. Morb Mortal Wkly Rep. el 21 de mayo de 2021;70(20):753-8.

14. Britton A. Effectiveness of the Pfizer-BioNTech COVID-19 Vaccine Among Residents of Two Skilled Nursing Facilities Experiencing COVID-19 Outbreaks - Connecticut, December 2020-February 2021. MMWR Morb Mortal Wkly Rep. 2021;70(20): 753-758. DOI: 10.15585/mmwr.mm7020e2

15. Bernal JL, Andrews N, Gower C, Robertson C, Stowe J, Tessier E, et al. Effectiveness of the Pfizer-BioNTech and Oxford-AstraZeneca vaccines on covid-19 related symptoms, hospital admissions, and mortality in older adults in England: test negative case-control study. BMJ. 13 de mayo de 2021;373:n1088. DOI: 10.1136/bmj.n1088

16. Ranzani OT, Hitchings M, Dorion M, D'Agostini TL, Paula RC de, Paula OFP de, et al. Effectiveness of the CoronaVac vaccine in the elderly population during a P.1 variant-associated epidemic of COVID-19 in Brazil: A test-negative case-control study. medRxiv. 28 de mayo de 2021. DOI: https://doi.org/ 10.1101/2021.05.19.21257472

17. Martínez-Baz I, Miqueleiz A, Casado I, Navascués A Trobajo-Sanmartin C, Burgui C, et al. Effectiveness of COVID-19 vaccines in preventing SARS-CoV-2 infection and hospitalisation, Navarre, Spain, January to April 2021. Eurosurveillance. 27 de mayo de 2021;26(21):2100438. DOI: 10.2807/1560-7917. ES.2021.26.21.2100438

18. Hitchings MDT, Ranzani OT, Torres MSS, Oliveira SB de, Almiron M, Said R, et al. Effectiveness of CoronaVac in the setting of high SARS-CoV-2 P.1 variant transmission in Brazil: A test-negative case-control study. medRxiv.7 de abril de 2021. DOI: https://doi. org/10.1101/2021.04.07.21255081 